\title{
Ausblick auf die zukünftige Entwicklung des Internationalen Immaterialgüterrechts in der Europäischen Union
}

\author{
Andreas Schwartze
}

\section{Einleitung}

Immaterialgüterrechte werden auch als ,geistiges Eigentum“ (international „Intellectual Property“ = IP) bezeichnet, ${ }^{1}$ wodurch eine Nähe zum Sachenrecht materieller Güter, seien sie beweglich oder unbeweglich, betont wird. ${ }^{2}$ Gemeinsam ist beiden Arten absoluter Rechte die Ausschließlichkeit, aus der sich die Abwehrrechte herleiten, sowie die Übertragbarkeit auf andere Personen. ${ }^{3}$

Auf der heutigen Tagung wurde das Urheberrecht behandelt: ${ }^{4}$ Es entsteht automatisch - aufgrund eines Realaktes der persönlichen geistigen Schöpfung - mit der Erstellung des Werkes. ${ }^{5}$ Dadurch sollen geistige oder künstlerische Leistungen vor unberechtigter Verwertung oder Veränderung geschützt werden. Es weist auch Verbindungen zum, ebenfalls absolut verstandenen, Persönlichkeitsrecht auf. ${ }^{6}$

\footnotetext{
${ }^{1}$ Vgl etwa Götting, Der Begriff des Geistigen Eigentums, GRUR 2006, 353 ff. Zur Entwicklung dieses Begriffs Peukert, in: Basedow/Hopt/Zimmermann (Hrsg), Handwörterbuch des Europäischen Privatrechts Bd. 1, Tübingen 2009.

${ }^{2}$ So dezidiert in Österreich aufgrund des weiten Eigentumsbegriffs in $\$ 285$ ABGB etwa Helmich in Kletecka/Schauer ABGB-ON § 285 Rz 1: ,unkörperliche Sachen“; Stabentheiner in Fenyves/ Kerschner/Vonkilch, Klang ${ }^{3} \S 285$ Rz 3: „Rechte“, Rz 4: „Vermögensrechte“. Fezer, Theorie der Funktionalität der Immaterialgüterrechte als geistiges Eigentum, GRUR 2016, 30 (33 f.), spricht insoweit von „Funktionseigentum“.

${ }^{3}$ Dazu näher Schöneich, Der Begriff der Dinglichkeit im Immaterialgüterrecht, Baden-Baden 2016, 175 ff., 195 ff.

${ }^{4}$ Büchele, in diesem Band Kap. 5.

${ }^{5}$ So $§ 10$ UrhG-AT, § 2 UrhG-DE, Art 6 lda-IT.

${ }^{6}$ Insbesondere im Bereich des sogenannten Urheberpersönlichkeitsrechts, wie in $\S \S 12-14$ Ur-
}

\author{
A. Schwartze $(\square)$ \\ Institut für Zivilrecht, Innsbruck, Österreich \\ E-Mail: Andreas.Schwartze@uibk.ac.at
}

(C) Der/die Autor(en) 2021

S. Laimer, C. Perathoner (Hrsg.), Italienisches, europäisches und internationales Immaterialgüterrecht, Bibliothek des Wirtschaftsrechts 1, https://doi.org/10.1007/978-3-662-62179-0_13 
Daneben wurde zum Patentrecht, ${ }^{7}$ zum Markenrecht ${ }^{8}$ sowie auch zum Geschmacksmusterrecht bzw Designrecht ${ }^{9}$ vorgetragen: Diese Ausschließlichkeitsrechte entstehen erst mit der Eintragung in ein spezielles Register. ${ }^{10}$ Sie sollen für technische oder wirtschaftliche Leistungen Schutz vor unbefugter Verwendung bieten. Aufgrund ihrer Bedeutung im unternehmerischen Bereich werden derartige property rights als ,gewerbliche Schutzrechte "11 bezeichnet.

$\mathrm{Zu}$ allen diesen Bereichen gibt es in den verschiedenen Rechtsordnungen spezielle Vorschriften. ${ }^{12}$ In ihnen werden vor allem die Entstehung, und damit die Erzeugung des subjektiven Rechts durch staatliche Verleihung, teilweise aber auch die Übertragung sowie die Haftung bei Verletzung dieser Rechte geregelt. Solange die Staaten in diesem Bereich souverän sind und autonom Recht setzen, gelten diese unterschiedlichen Regelungen allein in dem jeweiligen Staatsgebiet, es herrscht also das bereits auf dieser Tagung angesprochene Territorialitätsprinzip. ${ }^{13}$ Dies führt im Bereich des geistigen Eigentums zu einem Flickenteppich von weltweit mehr als 150 Immaterialgüterrechtsordnungen, ${ }^{14}$ deren Schutz auf das jeweilige Staatsgebiet beschränkt wird.

Da die Verbreitung von geistigen Schöpfungen notwendigerweise zu einem faktischen Kontrollverlust des Rechteinhabers führt, weil damit die Nutzungsmöglichkeit durch Andere eröffnet wird, ist angesichts der nicht auf ein Territorium beschränkbaren Öffentlichkeit ein grenzüberschreitender Schutz sinnvoll. Dieser kann entweder auf der Anwendung eines der nationalen Schutzrechte basieren, womit das Internationale Privatrecht ins Spiel kommt, oder aus einem überstaatlichen Immaterialgüterrecht abgeleitet werden, welches die Rechtsvereinheitlichung durch internationale Organisationen erfordert.

Im Folgenden werde ich diese beiden Bereiche des Internationalen Immaterialgüterrechts insbesondere mit Blick auf die Aktivitäten der Europäischen Union darstellen.

hG-DE sowie §§ 19-21 UrhG-AT; vgl etwa Fromlowitz, Das Urheberpersönlichkeitsrecht und das allgemeine Persönlichkeitsrecht, Bern 2013; Ubertazzi, Das EU-Reglement über die Urheberpersönlichkeitsrechte, GRUR Int 2018, 110; Toms in Kucsko/Handig, urheber.recht ${ }^{2} \S 19$ UrhG $\mathrm{Rz} 5 \mathrm{ff}$.

${ }^{7}$ Ferretti/Zito, in diesem Band Kap. 2.

${ }^{8}$ Perathoner, in diesem Band Kap. 1.

${ }^{9}$ Ferretti/Zito, in diesem Band Kap. 3; Steichele, in diesem Band Kap. 4.

${ }^{10}$ Allerdings kann ein Markenschutz in manchen Rechtsordnungen auch durch Benutzung und Erwerb von Verkehrsgeltung entstehen, so etwa § $4 \mathrm{Nr} 2$ MarkenG-DE.

${ }^{11}$ So etwa das Handbuch von Lendvai, Gewerbliche Schutzrechte, 7. Aufl., Köln 2017.

${ }^{12}$ Rechtsvergleichend dazu DACH - Europäische Anwaltsvereinigung, Gewerbliche Schutzrechte, Zürich 2011.

${ }^{13} \mathrm{Vgl}$ etwa Mestmäcker/Schweitzer, Europäisches Wettbewerbsrecht, 3. Aufl., München 2014, $\S 28 \mathrm{Rz} 3 \mathrm{f}$.

${ }^{14}$ Die jeweilige Rechtslage in den 193 WIPO-Mitgliedstaaten kann man auf der Website der WIPO unter https://wipolex.wipo.int/en/main/legislation nachvollziehen (Stand 14.05.2020). 


\section{Das Internationale Privatrecht für Immaterialgüter}

Das Internationale Immaterialgüterrecht im engeren Sinne erfasst allein grenzüberschreitende Sachverhalte und enthält Normen zur Bestimmung des jeweils anzuwendenden Rechts, im ergänzenden Bereich des Internationalen Prozessrechts solche zur (internationalen) Zuständigkeit der Gerichte sowie zur Anerkennung und Vollstreckung von (ausländischen) Urteilen. Auch diesen Rechtsbereich des Internationalen Privatrechts regeln die Staaten grundsätzlich eigenständig und durchaus in unterschiedlicher Weise - so etwa Italien in seinem IPR-Gesetz (IPRG-IT) von 1995, ebenso Österreich in seinem IPR-Gesetz (IPRG-AT) von 1979 und Deutschland im zweiten Kapitel des Einführungsgesetzes zum Bürgerlichen Gesetzbuch (EGBGB), Art 3 ff. Dadurch kommt es, je nach den am Gerichtsstand anzuwendenden IPR-Bestimmungen, unter Umständen zu unterschiedlichen Entscheidungen über das anzuwendende Recht. Um zu gleichen Ergebnissen zu gelangen, ist daher innerhalb der EU eine Vereinheitlichung des Internationalen Privatrechts auch für das Immaterialgüterrecht anzustreben, die in anderen großen Bereichen des Kollisionsrechts bereits erreicht ist. ${ }^{15}$

Um zu den zutreffenden Verweisungsnormen, ob im nationalen oder im Unionsrecht, zu gelangen, muss allerdings danach differenziert werden, welche materielle Rechtsfrage zu entscheiden ist.

Im Kern geht es meist um die Verletzung eines Immaterialgüterrechts, so dass Schadensersatzansprüche geltend gemacht werden. ${ }^{16}$ Dieses Thema ist heute bereits kollisionsrechtlich behandelt worden, ${ }^{17}$ ebenfalls unter den Aspekten des Gerichtsstandes ${ }^{18}$ sowie des materiellen Rechts. ${ }^{19}$ Hier ist nur noch einmal darauf hinzuweisen, dass dieser Bereich durch Unionsrecht bereits vereinheitlicht ist, so dass alle Gerichte innerhalb der EU (mit Ausnahme von Dänemark) nach der Rom IIVerordnung, konkret deren Art 8, zu entscheiden haben und damit in vergleichbaren Fällen dasselbe nationale Deliktsrecht anwenden. ${ }^{20}$ Gerichte außerhalb der EU ziehen dagegen weiterhin ihre eigenen Verweisungsnormen heran, so ist etwa in der Schweiz Art 110 Abs 1 IPRG-CH, allerdings mit inhaltlich gleicher Regelung wie nach der Rom II-VO, ausschlaggebend. Außerdem ist vorher innerhalb der EU der Gerichtsstand einheitlich nach der Brüssel Ia-VO zu ermitteln, Art 7 Zff 2, so dass

\footnotetext{
${ }^{15}$ Vor allem durch die Rom Verordnungen I - III, die EU-Erbrechtsverordnung sowie die beiden Güterrechtsverordnungen. Zu den aktuellen Entwicklungen vgl Mansel/Thorn/Wagner, Europäisches Kollisionsrecht 2018: Endspurt!, IPRax 2019, 85, sowie die vorangegangenen Jahresberichte.

${ }^{16} \mathrm{Zu}$ diesen aus österreichischer Sicht ausführlich Schacherreiter, Bereicherung und Schadenersatz im Immaterialgüterrecht, Wien 2018, S 39 ff.

${ }^{17}$ Laimer, in diesem Band Kap. 8.

${ }^{18}$ Kindler, in diesem Band Kap. 7.

${ }^{19}$ Christandl, in diesem Band Kap. 9.

${ }^{20} \mathrm{Vgl}$ Laimer, in diesem Band Kap. 8, bei Fn 21.
} 
auch keine unterschiedlichen Entscheidungen über die internationale Zuständigkeit vorkommen sollten. ${ }^{21}$

Als Vorfrage ist jedoch regelmäßig zu prüfen, ob das in Rede stehende Immaterialgüterrecht wirksam entstanden ist, welchen Inhalt es hat und ob es mittlerweile erloschen ist. Nach welchem Recht das zu entscheiden ist, richtet sich nach eigenen Kollisionsnormen, die sich in den jeweiligen nationalen Rechtsordnungen des Gerichtsortes finden, denn dieser Bereich des IPR ist noch nicht innerhalb der EU vereinheitlicht. So stellt Italien in Art 54 IPRG-IT etwa auf das Schutzlandprinzip ab, nach dem das Recht des Staates anzuwenden ist, für den bzw für dessen Gebiet der Schutz beansprucht wird. ${ }^{22}$ Obwohl in Österreich § 34 Abs 1 PRG-AT (,am Ort der Benützung- oder Verletzungshandlung") eher auf die international-deliktsrechtliche Tatortregel abgestellt wird, gelangt man zu sehr ähnlichen Ergebnissen, ${ }^{23}$ ebenso in Deutschland, wo auch ohne ausdrückliche Regelung gewohnheitsrechtlich an das Recht des Schutzlandes angeknüpft wird, ${ }^{24}$ und häufig auch außerhalb der EU, wie in der Schweiz nach Art 110 Abs 1 IPRG-CH (,,... Recht des Staates, für den der Schutz der Immaterialgüter beansprucht wird"). ${ }^{25}$ Eine andere Gruppe von Mitgliedstaaten, wie Griechenland, Portugal und Rumänien, knüpft zumindest im Urheberrecht dagegen an das Ursprungsland an, wo das Werk entstanden ist bzw wo der Urheber des Werks seinen Wohnsitz hat. ${ }^{26}$ Aufgrund dieser national unterschiedlichen Anknüpfungspunkte sowie Differenzen bei deren Auslegung wäre auch in diesem Bereich eine Vereinheitlichung innerhalb der EU wünschenswert. Diese sollte möglichst das Schutzlandprinzip verwenden, um in Übereinstimmung mit dem für die schadensrechtliche Betrachtung einschlägigen Art 8 Rom II-VO zur Anwendung ein und desselben Rechts zu gelangen. Dies gilt umso mehr, als eine Rechtswahl durch die Parteien weder für den Bestand des Schutzrechtes ${ }^{27}$ noch nach Art 8 (3) Rom II-Verordnung ${ }^{28}$ zulässig ist.

Weiterhin kann es in einem Immaterialgüterrechtsstreit um die Übertragung des geistigen Eigentums auf eine andere Person gehen, also etwa um einen Lizenzvertrag. Das auf diesen Vertrag anzuwendende Recht folgt wiederum eigenen Kollisionsnormen, welche allerdings in der EU ebenfalls vereinheitlicht sind. Die mitgliedstaatlichen Gerichte müssen hier das anwendbare Recht nach der für ver-

\footnotetext{
${ }^{21}$ Aufgrund des für grenzüberschreitende Schadensfälle geltenden Ubiquitätsprinzips sind jedoch unter Umständen die Gerichte verschiedener Staaten zuständig, vgl Kindler, in diesem Band, Kap. 7 (3.1., 3.4.4.).

${ }^{22} \mathrm{Vgl}$ Laimer, in diesem Band Kap. 8, Fn 16. Näher dazu Eccher in Christandl/Eccher/Gallmetzer/ Laimer/Schurr, Handbuch Italienisches Internationales Privatrecht, 2019, Rz 7/22.

${ }^{23}$ Nach den Gesetzesmaterialien sowie einheitlicher Lehre und Rspr ist ebenfalls auf das Recht des Schutzlandes abzustellen, Verschraegen in Rummel $\mathrm{ABGB}^{3}$ § 34 IPRG Rz 9.

${ }^{24}$ Drexl in MüKo/BGB ${ }^{7}$ IPR II Teil 8 Rz 198.

${ }^{25} \mathrm{Vgl}$ Laimer, in diesem Band Kap. 8, bei Fn 13.

${ }^{26} \mathrm{Vgl}$ Drexl in MüKo/BGB ${ }^{7}$ IPR II Teil 8 Rz 165.

${ }^{27}$ So etwa für Art 34 AT-IPRG, vgl Verschraegen in Rummel ABGB ${ }^{3} \S 34$ IPRG Rz 8.

${ }^{28}$ Anders Art 110 (2) CH-IPRG, der die Rechtswahl der lex fori nach Eintritt des schädigenden Ereignisses zulässt, vgl Th.Richter, Parteiautonomie im internationalen Immaterialgüterrecht, Tübingen $2017,155 \mathrm{ff}$.
} 
tragliche Schuldverhältnisse geltenden Rom I-Verordnung bestimmen. Da es keine spezielle Verweisungsnorm für diesen Vertragstyp gibt (obwohl eine solche im VO-Entwurf vorgesehen war, nämlich der gewöhnliche Aufenthalt des Lizenzgebers als in der Regel demjenigen, der die charakteristische Leistung erbringt), ${ }^{29}$ ist mangels einer Rechtswahl nach Art 3 Rom I-VO gem Art 4 Abs 2 Rom I-VO das Recht am gewöhnlichen Aufenthalt derjenigen Vertragspartei anzuwenden, welche die charakteristische Leistung erbringt, was im Regelfall eben der Lizenzgeber sein dürfte. ${ }^{30}$ Die auf diese Weise ermittelte Rechtsordnung erfasst jedoch allein das Verpflichtungsgeschäft, während die Übertragung des Immaterialgüterrechts im Wege eines Verfügungsgeschäfts wiederum dem nach den unterschiedlichen nationalen IPR-Regelungen für das Schutzrecht selbst anzuwendenden Recht unterliegt, meist also, wie oben dargestellt, ${ }^{31}$ dem Recht des Schutzlandes.

\section{Rechtsangleichung und Rechtsvereinheitlichung des Immaterialguiterrechts}

Die Verweisung auf eine nationale Rechtsordnung unter Anwendung des Internationalen Privatrechts ist allerdings nicht erforderlich, wenn in den beteiligten Staaten identische Regelungen gelten, also internationales Einheitsrecht zur Anwendung kommt. Im Folgenden sollen europäische rechtsangleichende und rechtsvereinheitlichende Maßnahmen im Bereich des geistigen Eigentums dargestellt werden.

\subsection{Gesamteuropäische Instrumente}

Eine erste, wenn auch nur strukturelle, Vereinheitlichung hat der seit 1949 bestehende Europarat, dem derzeit 47 europäische Staaten angehören, mit dem ,Straßburger Abkommen über die internationale Patentklassifikation“ von 1954, ${ }^{32}$ das ein einheitliches System mit identischen Gruppen zur Klassifizierung von Patenten für Erfindungen vorsah, vorgenommen, welches dann im Jahre 1971 in die Verwaltung der WIPO (World Intellectual Property Organisation) ${ }^{33}$ überführt wurde. Auf dieser Grundlage, die regelmäßig aktualisiert wird, erfolgt die Internationale Patentklassifikation in mehr als 100 Staaten. Eine weitere Rechtsharmonisierung erfolgte durch das „Übereinkommen zur Vereinheitlichung gewisser Begriffe des materiellen

\footnotetext{
${ }^{29}$ Dazu Stimmel, Die Beurteilung von Lizenzverträgen unter der Rom I-Verordnung, GRUR Int 2010, 783 (784).

${ }^{30}$ Spickhoff in BeckOK ${ }^{53}$ VO (EG) 593/2008 Art 4 Rz 71.

${ }^{31}$ Sh oben bei Fn 22.

${ }^{32}$ European Convention on the International Classification of Patents for Inventions, ETS No. 017,

${ }^{33} \mathrm{Vgl}$ das Übereinkommen zur Errichtung der Weltorganisation für geistiges Eigentum von 1967, mit der diese Unterorganisation der UNO mit Sitz in Genf errichtet wurde.
} 
Rechts der Erfindungspatente“ von 1963, ${ }^{34}$ wenn auch räumlich stark begrenzt, da es nur von 13 Staaten, darunter Deutschland, Italien und der Schweiz, dagegen etwa nicht von Österreich, ratifiziert wurde. Mit diesem Instrument sollten die Bedingungen vereinheitlicht werden, die für die Erteilung eines Patents erforderlich sind, und die Kriterien bestimmt werden, die von den Gerichten bei der Festlegung des Schutzbereichs des Patents zu beachten sind.

Seit 1977 konnte dann ein mittlerweile in 38 Staaten geltendes einheitliches Europäisches Patent beantragt werden. Das wurde auf der Grundlage des 1973 abgeschlossenen Europäischen Patentübereinkommens (EPÜ) ${ }^{35}$ möglich, dem außer der EU, deren Mitgliedstaaten sämtlich beteiligt sind, weitere 10 Nicht-EU-Staaten beigetreten sind, darunter auch die Schweiz und Liechtenstein. Dieses beim Europäischen Patentamt (EPA) zu beantragende Schutzrecht wirkt allerdings in den beteiligten Staaten wie deren jeweiliges nationales Patent (Art 64 EPÜ). Damit führt es nur zu einem Bündel nationaler Rechte, welche in ihrem Bestehen voneinander unabhängig sind. Allerdings ist ihr abgegrenzter Schutzbereich für die eigenständigen Patentansprüche dann doch wieder einheitlich nach Art 69 EPÜ auszulegen. Weil die Wirkungen nationaler und übernationaler Patente damit letztlich übereinstimmen, haben die meisten Vertragsstaaten im Wege einer freiwilligen Harmonisierung ihr innerstaatliches Patentrecht an das EPÜ angepasst. ${ }^{36}$

\subsection{Instrumente der Europäischen Union}

Innerhalb der EU ist für die Schaffung sekundären Unionsrechts zunächst Art 345 AEUV zu beachten, nach dem die Eigentumsordnung der Mitgliedstaaten und damit auch das System ihres Immaterialgüterrechts unberührt bleiben - es könnte bei der Erzeugung derartiger Herrschaftsrechte auf Unionsebene also die Kompetenz fehlen. Mit dem Vertrag von Lissabon wurde ab Dezember 2009 jedoch eine eigene Kompetenzgrundlage für ,europäische Rechtstitel über einen einheitlichen Schutz der Rechte des geistigen Eigentums“ sowie ,zentralisierte Zulassungs- und Kontrollregelungen“ (Art 118 Abs 1 AEUV) in die EU-Verträge aufgenommen.

Bereits seit 1986 wurden jedoch Richtlinien erlassen, die zur Harmonisierung der mitgliedstaatlichen Immaterialgüterrechte führen, mithin die zwischen ihnen bestehenden Regelungsunterschiede abbauen sollen. ${ }^{37}$ Sie wurden auf die allgemeine

\footnotetext{
${ }^{34}$ Convention on the Unification of Certain Points of Substantive Law on Patents for Invention, ETS No. 047.

${ }^{35}$ Mittlerweile in der Fassung nach der „Akte zur Revision des Übereinkommens über die Erteilung europäischer Patente“, abgedruckt in GRUR Int 2001, 309, dazu Adam/Grabinski in Benkard, Europäisches Patentübereinkommen ${ }^{3}$, Rz 23 ff., als „EPÜ 2000“, verfügbar unter https://www.epo. org/law-practice/legal-texts/html/epc/2016/d/EPC_conv_20200401_de_20200320.pdf.

${ }^{36}$ In Deutschland etwa durch das PatentG 1980.

${ }^{37}$ Einen Gesamtüberblick gibt Tattay, Entwicklungsgeschichte der Rechte des geistigen Eigentums in der Europäischen Union, GRUR Int 2013, 2012.
} 
Kompetenz zur Harmonisierung zugunsten einer Förderung des Binnenmarktes gestützt (heute Art 114, 115 AEUV). Inhaltlich liegt der Schwerpunkt im Urheberrecht mit verwandten Schutzrechten, denn diesen erfassen elf Richtlinien ${ }^{38}$ (z. B. die Richtlinie zur Harmonisierung der Schutzdauer des Urheberrechts und bestimmter verwandter Schutzrechte 2011, ${ }^{39}$ oder die Richtlinie zur Harmonisierung bestimmter Aspekte des Urheberrechts und der verwandten Schutzrechte in der Informationsgesellschaft, sogenannte „InfoSoc-Richtlinie“, 2001, ${ }^{40}$ die mittlerweile in der lange umstrittenen jüngsten Richtlinie über das Urheberrecht und die verwandten Schutzrechte im digitalen Binnenmarkt 2019, ${ }^{41}$ welche vor allem das Urhebervertragsrecht harmonisiert, aufgegangen ist),. Dagegen beziehen sich auf den Markenschutz nur zwei Richtlinien (Erste Richtlinie zur Rechtsangleichung der Rechtsvorschriften der Mitgliedstaaten über die Marken von $1989 ;{ }^{42}$ Richtlinie zur Angleichung der Rechtsvorschriften der Mitgliedstaaten über die Marken von 2015), ${ }^{43}$ mit jeweils einer speziellen Richtlinie ergänzt um den Designschutz (Richtlinie über den rechtlichen Schutz von Mustern und Modellen 1998) ${ }^{44}$ sowie den Schutz der dreidimensionalen Strukturen von Mikro-Chips (Richtlinie über den Schutz der Topografien von Halbleitererzeugnissen 1986). ${ }^{45}$ Im Bereich des Patentrechts wurde allein eine Richtlinie über den rechtlichen Schutz biotechnologischer Erfindungen von $1998^{46}$ in Kraft gesetzt. Zur Harmonisierung der zivilrechtlichen Rechtsfolgen von Verletzungen auch nationaler Schutzrechte soll die Richtlinie über die Durchsetzung der Rechte des geistigen Eigentums von $2004{ }^{47}$ beitragen, die vereinheitlichte Durchsetzungsregelungen im Urheber-, Patent- und Markenrecht sowie für andere geistige Eigentumsrechte vorsieht; sie wirkt sich mit ihren Bestimmungen vor allem zur Informationsgewinnung im Vorfeld eines Verfahrens auch auf das Zivilprozessrecht der Mitgliedstaaten aus. Im Bereich der Richtlinien muss das Recht sämtlicher Mitgliedstaaten entsprechend angepasst werden, einheitliche Regelungen oder gar Unions-Schutzrechte werden dadurch jedoch nicht geschaffen - somit bleibt die territoriale Fragmentierung erhalten.

\footnotetext{
${ }^{38}$ Einen Überblick bietet Stollwerck in BeckOK UrhR, 26. Ed (15.10.2019), UrhG Europäisches Urheberrecht Rz 3 ff. Vgl auch Metzger, Rechtsfortbildung im Richtlinienrecht, ZEuP 2017, 836 (838-840).

${ }^{39}$ RL 2011/77/EU, ABl 2011, L 265/1. Diese ersetzt die Richtlinie 2006/116/EG, ABl 2006, L 372/12, welche wiederum die Richtlinie 93/98/EWG, ABl 1993, L 290/9, aufgehoben hatte.

${ }^{40}$ RL 2001/29/EG, AB1 2001, L 167/10.

${ }^{41}$ RL 2019/790/EU, AB1 2019, L 130/92.

${ }^{42}$ RL 89/104/EG, ABl 1989, L 40/1, in kodifizierter Form als RL 2008/95/EG, AB1 2008, L 299/25.

${ }^{43}$ RL 2015/2436/EU, ABl 2015, L 336/1. Damit wurde die eben erwähnte RL 2008/95/EG, AB1 2008, L 299/25, ersetzt.

${ }^{44}$ RL 98/71/EG, ABl 1998, L 289/28.

${ }^{45}$ RL 87/54/EWG, AB1 1987, L 24/36.

${ }^{46}$ RL 98/44/EG, AB1 1998, L 213/13. Dazu etwa Kienle, Die neue EU-Richtlinie zum Schutz biotechnologischer Erfindungen, WRP 1998, 692.

${ }^{47}$ RL 2004/48/EG, AB1 2004, L 195/16. Einen Überblick geben Frey/Rudolph, EU-Richtlinie zur Durchsetzung der Rechte des geistigen Eigentums, ZUM 2004, 522.
} 
Im Bereich des Markenrechts hat die EU mit der Unionsmarken-Verordnung von $2015,{ }^{48}$ der früheren Gemeinschaftsmarken-Verordnung von $1994,{ }^{49}$ eine aufgrund einer Anmeldung entweder bei einer nationalen Behörde oder beim Amt der Europäischen Union für Geistiges Eigentum (EUIPO) in Alicante in allen ihren Mitgliedstaaten einheitlich geltende Marke geschaffen, die neben die nationalen Marken tritt. Seit 2002 gilt eine gleichartige Regelung für das Gemeinschaftsgeschmacksmuster, ${ }^{50}$ bereits seit 1992 auch für den Schutz geografischer Herkunftsangaben für Lebensmittel und Agrarerzeugnisse. ${ }^{51}$ Alle diese Unionsschutzrechte entfalten eine einheitliche Wirkung in sämtlichen Mitgliedstaaten, werden einheitlich erteilt, beendet und können, mit Ausnahme der geografischen Herkunftsangaben, einheitlich übertragen werden. Allerdings ersetzen sie nicht die nationalen Schutzrechte, sondern bestehen neben diesen, so dass sie sie ergänzen. Ein sehr ähnliches Modell wird etwa im Bereich des Europäischen Gesellschaftsrechts verfolgt, indem auf der Unionsebene eigenständige Kooperationsformen neben den nationalen Gesellschaftstypen zur Wahl gestellt werden, wie die Europäische Aktiengesellschaft/Societas Europaea (SE) oder die Europäische Genossenschaft, ${ }^{52}$ und auch im Europäischem Vertragsrecht wurde mit dem zusätzlich zum jeweiligen innerstaatlichen Vertragsrecht zur Verfügung stehenden Vorschlag für ein Gemeinsames Europäisches Kaufrecht (GEKR) dieser Weg eingeschlagen. ${ }^{53}$

International wird eine ähnliche Wirkung durch eine einzige Eintragung über das Madrider Markenabkommen von 1891 in der Fassung von $1967^{54}$ (sowohl in Italien wie in Österreich, ebenso in Deutschland, in Kraft, insgesamt in über 50 Ländern) erreicht, allerdings bündelt diese Internationale Marke nur nationale Marken nach dem jeweiligen nationalen Recht, ohne dass eine Marke mit einheitlicher Schutzwirkung entsteht.

Es fehlt jedoch vor allem an einem einheitlichen Schutz der Urheberrechte, obwohl eine entsprechende Verordnung mittlerweile, wie oben erwähnt, gem Art 118 (1) AEUV in der Kompetenz der EU läge. Bereits im Jahre 2010 wurde von einer Gruppe europäischer Akademiker der Entwurf eines einheitlichen europäischen Ur-

\footnotetext{
${ }^{48}$ VO 2015/2424/EU, ABl 2015, L 341/88.

${ }^{49} \mathrm{VO}$ 40/94/EG, AB1 1994, L 11/1.

${ }^{50}$ VO 6/2002/EG, AB1 2002, L 3/1. Vgl etwa Schlötelburg, Das neue Europäische Geschmacksmusterrecht, MittdtPatA 2002, 70.

${ }^{51}$ Mittlerweile VO 1151/2012, AB1 2012, L 343/1, welche die VO 510/2006/EG, AB1 2006, L 93/12 ersetzte, die wiederum an Stelle der VO 2081/92/EG, ABl 1992. L 208/1; näheres bei Faßbender/Herbrich, Geografische Herkunftsangaben im Spannungsfeld von nationalem und europäischem Recht, GRUR Int 2014, 765. Zu derartigen Ursprungsbezeichnungen im CETA-Handelsabkommen zwischen der EU und Kanada Miribung, in diesem Band Kap. 6.

${ }^{52} \mathrm{Vgl}$ dazu etwa Kalss/Klampfl, Europäisches Gesellschaftsrecht, 2015, Rn 550 ff.; Habersack/ Verse, Europäisches Gesellschaftsrecht ${ }^{5}, 2019,497$ ff.

${ }^{53}$ Wendehorst, Der Anwendungsbereich eines Gemeinsamen Europäischen Kaufrechts, AnwBl 2014, 345; dazu auch Schwartze, § 4 Rechtsvergleichung, in: Riesenhuber (Hrsg.), Europäische Methodenlehre ${ }^{3}$, 2015, 53 (61 f.).

${ }^{54}$ Madrider Abkommen über die internationale Registrierung von Marken, https://transpatent.com/ archiv/194mma/mma.html.
} 
heberrechtsgesetzes (European Copyright Code) ${ }^{55}$ vorgestellt und das Europäische Parlament (EP) hat 2014 ein EU-Urheberrecht vorgeschlagen. ${ }^{56}$ Die Kommission hat sich jedoch in ihrer Strategie für den digitalen Binnenmarkt auf die Weiterentwicklung der Urheberrechts-Richtlinien konzentriert, was zum Erlass der Urheberrechts-Richtlinie von $2019^{57}$ führte. Die Koexistenz identischer Rechte in allen Mitgliedstaaten, wie bei den gewerblichen Schutzrechten, erscheint bei dem formlos, ohne Registrierung, entstehenden Urheberrecht jedenfalls nicht zielführend. Stattdessen wäre es sinnvoll, die urheberrechtsrelevanten Nutzungshandlungen einheitlich zu definieren.

\section{Private Regulierung}

Zum Schluss noch einige Überlegungen zu den Gestaltungsmöglichkeiten im Bereich des internationalen Immaterialgüterrechts im Rahmen der Privatautonomie.

Zwar können die Parteien, wenn auch gemeinsam, sich kein Immaterialgüterstatut aussuchen, da die Rechtswahlfreiheit im Internationalen Privatrecht insoweit beschränkt ist, auch in Bezug auf die aus dem Deliktsrecht abgeleiteten Verletzungsfolgen, wie ausdrücklich durch Art 8 Abs 3 Rom II-VO angeordnet. ${ }^{58}$ Die Schaffung eines Schutzrechts qua transnationalem Gewohnheitsrecht (etwa der lex mercatoria) entfällt aufgrund des numerus clausus der Eigentumsrechte ${ }^{59}$ ebenfalls. Immerhin können jedoch einheitliche Nutzungsbedingungen vereinbart werden, so z. B. der Verzicht auf Rechte, Einwilligungen in bestimmte Nutzungen oder umfassendere Lizenzverträge, für die das insoweit anzuwendende Vertragsstatut durchaus einer Rechtswahl offen steht. ${ }^{60}$ Regelmäßig werden diese Abreden jedoch nur zweiseitig wirken, also allein die beiden Parteien erfassen. Durch die Verbindung zu einem Vertragsnetz ${ }^{61}$ oder zu einem Netzwerkvertrag können sie jedoch auf eine Vielzahl von Akteuren erstreckt werden.

Hinsichtlich der Streitentscheidung können die Parteien eine Schiedsgerichtsvereinbarung treffen, so dass statt eines staatlichen Gerichts von den Beteiligten

\footnotetext{
${ }^{55} \mathrm{https}: / /$ www.ivir.nl/copyrightcode/european-copyright-code/.

${ }^{56}$ Rechtsausschuss, Entwurf eines Berichts über die Umsetzung der RL 2001/29/EC zur Harmonisierung bestimmter Aspekte des Urheberrechts und der verwandten Schutzrechte in der Informationsgesellschaft (2014/2256[INI], A8 - 0209/2015 (Berichterstatterin: Julia Reda).

${ }^{57}$ Sh oben Fn 41.

${ }^{58}$ Ebenso zuvor durch die Rechtsprechung etwa nach österreichischem sowie deutschem Kollisionsrecht, vgl Th. Richter, Parteiautonomie im Internationalen Immaterialgüterrecht, 2017, 100-106, 116.

${ }^{59}$ Dazu ausführlich etwa M. Schroeder, Numerus clausus der Immaterialgüterrechte?, 2017.

${ }^{60}$ Sh oben nach Fn 29.

${ }^{61}$ Dazu gehören etwa Patentpools, Verwertungsgesellschaften oder auch Open Source-Zugänge. Grundlegend dazu Teubner, Netzwerk als Vertragsverbund, 2004.
} 
ausgewählte Schiedsrichter tätig werden, wodurch die Vervielfachung der Gerichtsstände aufgrund des Ubiquitätsprinzips ${ }^{62}$ vermieden wird.

Auf eine freiwillige Beachtung durch die Betroffenen setzen zudem Verhaltenskodizes, welche als soft law ${ }^{63}$ vor allem dann Bedeutung gewinnen, wenn die Beteiligten sich durch eine Selbstverpflichtung an sie binden. Der dem Recht eigene (staatliche) Zwang zu seiner Durchsetzung, dem sowohl Verträge als auch die eben angesprochenen Schiedssprüche unterworfen sind, fehlt hier allerdings, weshalb allein sozialer Druck zur Einhaltung der in codes of conduct aufgestellten Regeln beitragen kann.

Mit den angeführten Instrumenten wird es den am geistigen Eigentum, vor allem an dessen Nutzung, Interessierten ermöglicht, in einem - wenn auch begrenzten Maße eine Harmonisierung des internationalen Immaterialgüterrechts „,von unten“ ( bottom up) durchzusetzen. Auf weitere Maßnahmen staatlicher bzw überstaatlicher Stellen „von oben“ (top down) kann gleichwohl nicht verzichtet werden.

\footnotetext{
${ }^{62}$ Sh oben bei Fn 21.

${ }^{63}$ Dazu etwa Schwartze, Europäisierung des Zivilrechts durch „soft law“, in: Eger/H.-B.Schäfer (Hrsg.), Ökonomische Analyse der europäischen Zivilrechtsentwicklung, 2007, 130 ff.
}

Open Access Dieses Kapitel wird unter der Creative Commons Namensnennung 4.0 International Lizenz (http://creativecommons.org/licenses/by/4.0/deed.de) veröffentlicht, welche die Nutzung, Vervielfältigung, Bearbeitung, Verbreitung und Wiedergabe in jeglichem Medium und Format erlaubt, sofern Sie den/die ursprünglichen Autor(en) und die Quelle ordnungsgemäß nennen, einen Link zur Creative Commons Lizenz beifügen und angeben, ob Änderungen vorgenommen wurden.

Die in diesem Kapitel enthaltenen Bilder und sonstiges Drittmaterial unterliegen ebenfalls der genannten Creative Commons Lizenz, sofern sich aus der Abbildungslegende nichts anderes ergibt. Sofern das betreffende Material nicht unter der genannten Creative Commons Lizenz steht und die betreffende Handlung nicht nach gesetzlichen Vorschriften erlaubt ist, ist für die oben aufgeführten Weiterverwendungen des Materials die Einwilligung des jeweiligen Rechteinhabers einzuholen. 